\title{
SU-8 based microdevices to study self-induced chemotaxis in 3D microenvironments
}

\author{
Jose Maria Ayuso 1,2,3, Rosa Monge ${ }^{1,2,3}$, Guillermo A. Llamazares ${ }^{1,2,3}$, Marco Moreno $^{1,2,3}$, \\ Maria Agirregabiria ${ }^{4}$, Javier Berganzo ${ }^{5}$, Manuel Doblaré ${ }^{1,2,3}$, Iñaki Ochoa ${ }^{1,2,3 \dagger}$ and \\ Luis J. Fernández ${ }^{1,2,3+*}$ \\ ${ }^{1}$ Group of Structural Mechanics and Materials Modelling (GEMM), Centro de Investigación Biomédica en Red en \\ Bioingeniería, Biomateriales y Nanomedicina (CIBER-BBN), Zaragoza, Spain, ${ }^{2}$ Aragón Institute of Engineering Research (IJA), \\ University of Zaragoza, Zaragoza, Spain, ${ }^{3}$ Aragon Institute of Biomedical Research, Instituto de Salud Carlos III, Zaragoza, \\ Spain, ${ }^{4}$ MEMS/MST Department, Ikerlan S. Coop., Mondragón, Spain, ${ }^{5}$ Department of Design and Manufacturing \\ Engineering, University of Zaragoza, Zaragoza, Spain
}

\section{OPEN ACCESS}

Edited by:

Daniel J. Solis,

BioNano Genomics, USA

Reviewed by:

Eduardo Fernandez,

University Miguel Hernandez, Spain Verena Charwat,

University of Natural Resources and

Life Sciences, Austria

*Correspondence:

Luis J. Fernández,

Group of Structural Mechanics and Materials Modelling (GEMM), I3A,

Zaragoza University, Marinano

Esquillor, Zaragoza 50018, Spain luisf@unizar.es

${ }^{\dagger}$ Iñaki Ochoa and Luis J. Fernández have contributed equally to this work

Specialty section: This article was submitted to Translational Materials Science, a section of the journal Frontiers in Materials

Received: 21 November 2014 Accepted: 11 April 2015 Published: 04 May 2015

Citation: Ayuso JM, Monge R, Llamazares GA, Moreno $M$, Agirregabiria $M$, Berganzo J, Doblaré M, Ochoa I and Fernández LJ (2015) SU-8 based microdevices to study self-induced chemotaxis in $3 D$ microenvironments.

Front. Mater. 2:37.

doi: 10.3389/fmats.2015.00037
Tissues are complex three-dimensional structures in which cell behavior is frequently guided by chemotactic signals. Although starvation and nutrient restriction induce many different chemotactic processes, the recreation of such conditions in vitro remains difficult when using standard cell culture equipment. Recently, microfluidic techniques have arisen as powerful tools to mimic such physiological conditions. In this context, microfluidic three-dimensional cell culture systems require precise control of cell/hydrogel location because samples need to be placed within a microchamber without obstruction of surrounding elements. In this article, SU-8 is studied as structural material for the fabrication of complex cell culture microdevices due to its good mechanical properties and sensor integration capacity. Moreover, SU-8 physical properties and their effect on a successful design for precise control of hydrogel location within microfluidic devices are studied. In particular, this manuscript presents a SU-8 based microdevice designed to create "self-induced" medium starvation, based on the combination of nutrient restriction and natural cell metabolism. Results show a natural migratory response toward nutrient source, showing how cells adapt to their own microenvironment modifications. The presented results demonstrate the SU-8 potential for microdevice fabrication applied to cell culture.

Keywords: SU-8 photoresist, microfluidic devices, capillarity, microfluidic cell culture, hydrogel confinement, self-induced chemotaxis, cell migration

\section{Introduction}

Living tissues are composed of embedded cells within a heterogeneous extracellular matrix, which plays a key role in three-dimensional cell migration and intercellular communication (Raines, 2000; Savino et al., 2004; Korpos et al., 2010; Franco and Muller, 2011). Several previous studies report that cell culture in three-dimensional systems produces multiple changes in cell behavior when compared to the use of classical two-dimensional systems (Baker and Chen, 2012; Huang et al., 2012; Breslin and O'Driscoll, 2013; Page et al., 2013). In migration, such behavioral differences are caused not only by the availability of a three-dimensional environment affecting cell migration but also by generation of chemotactic processes. For example, cancer cells have been reported to migrate and 
invade other tissues following chemotaxis induced by SDF-1, FGF, PDGF, or TGF $\beta$ (Roussos et al., 2011). Examples of chemotactic processes are angiogenesis (Guo et al., 2012), immune response (Nieto et al., 1997), and axon elongation during embryonic development (Bhattacharjee et al., 2010). Numerous reports have shown the influence of nutrient on cell migration (Chen et al., 2011). In this context, tumor cells often proliferate much faster than non-malignant cells, causing nutrient starvation in tumor microenvironments (Noman et al., 2011). These conditions trigger tumor cell migration and invasiveness, worsening patient prognosis (Nagelkerke et al., 2013). Therefore, there is increasing interest among the biological community in study cell response under these nutrient restricted conditions (Kim et al., 2013a; Zhang et al., 2013). Various strategies have been developed for the study of cell migration in response to chemotaxis. Perhaps one of the most well-known assays involves the use of a Boyden chamber, in which cells migrate through a polycarbonate membrane in response to chemoattractants (Falasca et al., 2011). However, as this system does not allow direct observation of cellular migration, alternative tools were developed such as the under-agarose gel (Mousseau et al., 2007), Zigmond chamber (Zigmond, 1977), Dunn chamber (Zicha et al., 1997), and Insall chamber assays (Muinonen-Martin et al., 2010). Although each of these assays has its own advantages, they generally struggle to maintain stable gradients for long periods of time (Keenan and Folch, 2008; Muinonen-Martin et al., 2010) and more important: the chemotaxis conditions are externally imposed. In all of these assays, study how cells response to their own nutrient consumption remains challenging. Recently, microfabrication and microfluidic technologies have arisen as interesting alternatives for creating high-performance cell culture systems (El-Ali et al., 2006; Kim et al., 2010; Lesher-Perez et al., 2013). In general, such devices possess a culture microchamber housing a hydrogel and lateral microchannels delimited by a series of pillars (Figure 1). These lateral microchannels are usually kept hydrogel-free to allow for medium perfusion (Huang et al., 2009; Farahat et al., 2012; Song et al., 2012). Depending on the experimental protocol, cells could either be embedded within the hydrogel or located in the lateral microchannels. Injecting different media on each lateral microchannel chemical gradients across the culture chamber can be imposed (Huang et al., 2009; Kothapalli et al., 2011; Farahat et al., 2012; Funamoto et al., 2012). The majority of recently

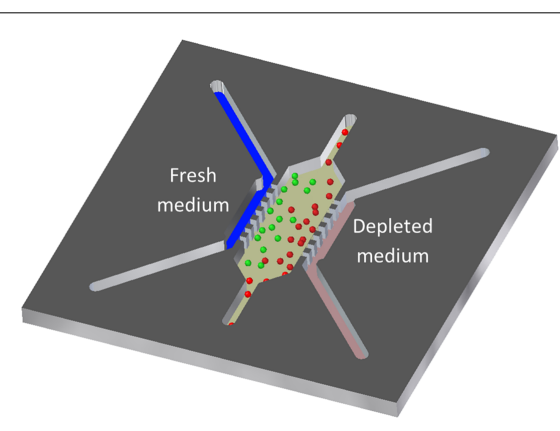

FIGURE 1 | The SU-8-based microdevice possesses a central culture microchamber and two lateral microchannels to enable medium flow. designed microfluidic devices are fabricated using polydimethylsiloxane (PDMS), which possesses excellent optical properties. However, using PDMS has some disadvantages, including poor mechanical properties (Jeon et al., 2011) and sensor integration is challenging. Here, we present a SU- 8 based microfluidic device for three-dimensional cell cultures capable to establish nutrient restricted conditions. SU-8 material was selected due to the wide range of sensors and actuators already reported to be available to be monolithically integrated: valves (Ezkerra et al., 2007; Calvo et al., 2011), pumps (Ezkerra et al., 2011), flow sensors (Vilares et al., 2010), microneedles (Fernández et al., 2009), polymerase chain reaction (PCR) systems (Verdoy et al., 2012), cell sorting devices (González et al., 2012), electrophoresis (Castaño-Álvarez et al., 2009), and Mach-Zender interferometer based sensors (Duval et al., 2012) among others. Additionally, SU-8 have been reported previously for cell culture applications (Kotzar et al., 2002; Nemani et al., 2013; Torrejon et al., 2013; Rigat-Brugarolas et al., 2014), showing some advantages over PDMS for the fabrication of cell cultures systems, including broader chamber design possibilities (Ni et al., 2009), good mechanical properties (Lorenz et al., 1997), and gas impermeability (Gerhardt and Betsholtz, 2005). Huang et al. described extensively that when using PDMS microdevices, the hydrogel location can be achieved creating a specific geometry based on a series of pillars and taking advantage of PDMS surface hydrophobicity (Huang et al., 2009). They also demonstrated how when hydrophilic material are used, hydrogel cannot be confined using the same geometry. As SU-8 is hydrophilic (Jokinen et al., 2012), those described designs are not applicable. Although SU-8 can be chemically treated to render its surface hydrophobic, this requires aggressive and dangerous chemical agents such as strong acids and hydrogen peroxide (Schumacher et al., 2008). In this study, a new design capable of confining hydrogels within hydrophilic microdevices was successfully developed and used with SU-8-based microfluidic devices. The biocompatibility of SU-8-based microdevices was evaluated at different cell densities and collagen concentration using the pre-osteoblasts cell line, MC3T3. Finally, cells were long-term cultured within the microdevice under two different conditions: (1) unrestricted conditions, medium was refreshed once a day through both lateral microchannels; (2) Restricted conditions, one lateral microchannel was sealed while continuous medium perfusion was enabled on the other lateral microchannel. After 1 week, in culture cell distribution within the central microchamber was analyzed, showing an intense self-induced chemotactic response under restricted conditions due to cell metabolism. Contrary to previous cell culture studies under artificially generated gradients (Zicha et al., 1997; Zimmermann et al., 2007; Bhattacharjee et al., 2010; Kim et al., 2013b, 2010; Chen et al., 2011; Funamoto et al., 2012) the proposed device and protocol allows the study of cell response under self-induced nutrient gradient conditions. The results presented in this paper validate $\mathrm{SU}-8$ based microfluidic devices as a novel and robust tool for future three-dimensional self-induced cell migration assays and allow studying cell behavior under nutrient starvation. In this article, this methodology is validated in the context of bone tissue repair, since blood vessels are absent in bone scaffolds and nutrient support is compromised. This is the reason why MC3T3 cells were selected. However, 
the described methodology could be applied in many different biological scenarios.

\section{Materials and Methods}

\section{Microfluidic Chip Fabrication}

Microfluidic devices were fabricated using SU-8 photolithography combined with an $\mathrm{SU}-8$ to $\mathrm{SU}-8$ bonding process (Blanco et al., 2004). Figure 2 summarizes the fabrication process, which yielded a stand-alone SU-8 microfluidic chip that was without any substrate material such as glass or silicon. The fabrication process was inspired by previously reported work describing the fabrication of SU-8 microneedles (Altuna et al., 2012, 2013). First, a polyimide film (Kapton) was temporarily bonded onto a Pyrex wafer (Figure 2A). Then, a $60 \mu \mathrm{m}$-thick layer of SU8 was spun onto the Kapton film and soft baked at $65^{\circ} \mathrm{C}$ for $30 \mathrm{~min}$. After the wafer was cooled down to room temperature, a spinning protocol for the deposition of a $20 \mu \mathrm{m}$-thick SU-8 layer was performed, followed by an additional soft bake. The total thickness of obtained SU-8 layers was $90 \mu \mathrm{m}$ instead of the expected $80 \mu \mathrm{m}$ (Figure 2B). This can be explained by the higher frictional forces present when layering on an SU-8 surface compared to Kapton. The wafer was then exposed to a $140 \mathrm{~mJ} / \mathrm{cm}^{2}$ dose of UV light filtered with a mask that defines the bottom layer of the device. The exposure was followed by a post-bake step which consisted of heating the wafer to $65^{\circ} \mathrm{C}$ for $15 \mathrm{~min}$. Three new depositions of SU-8 layers were then performed (60-, 60-, and $20-\mu \mathrm{m}$ thick), followed by corresponding soft bakes, producing a total height of $150 \mu \mathrm{m}$. The wafer was again exposed to UV light $\left(140 \mathrm{~mJ} / \mathrm{cm}^{2}\right)$ to pattern microchannels and microchambers, post baked (Figure 2C), immersed in SU-8 developer, and finally rinsed in DI $\mathrm{H}_{2} \mathrm{O}$ (Figure 2D).
An additional Pyrex wafer was processed with temporary bonding to a Kapton film (Figure 2E). The previously described spinning procedures and soft bake processes were applied to this wafer to obtain a $90-\mu \mathrm{m}$-thick SU-8 layer. Inlets and outlets were defined by standard photolithography using a $140 \mathrm{~mJ} / \mathrm{cm}^{2}$ dose of UV light. Then, a $150 \mu \mathrm{m}$-thick layer of SU-8 was deposited and patterned with the same mask used to obtain microchannels and microchambers on the first wafer (Figure $\mathbf{2 H}$ ). Both wafers were then aligned and bonded by heating to $90^{\circ} \mathrm{C}$ for 15 min under a pressure of 3 bar (Figure 2I). The weak adhesion between SU-8 and Kapton allowed the devices to be released manually (Figure 2J). A picture of a finished chip is illustrated in Figure 2K.

\section{Packaging Tool Fabrication}

In order to recreate restricted conditions, a regular flow of medium must be provided through the microdevice. A dedicated package was designed and fabricated to provide proper housing and hermetic connections to the microdevice inlets and outlets, allowing for automated cellular culture. The package body was made of polycarbonate to withstand cell culture humidity and temperature conditions. Flat bottom Upchurch fluidic connectors (Idex M644-03x) were used to reduce dead volume and provide a smooth fluid tube-to-package transition. The package is divided into two parts: a microdevice holder with an optical observation window, and an upper manifold cover that holds inlet sealing o-rings (Barnwell BS1806-001) and tube connectors. When the manifold cover is screwed down over the chip holder, the microdevice is sealed and connected to the tubes. Both parts were fabricated with precision machining, taking special care to avoid roughness on critical surfaces such as sealing elements, fluidic connectors, and o-ring grooves.

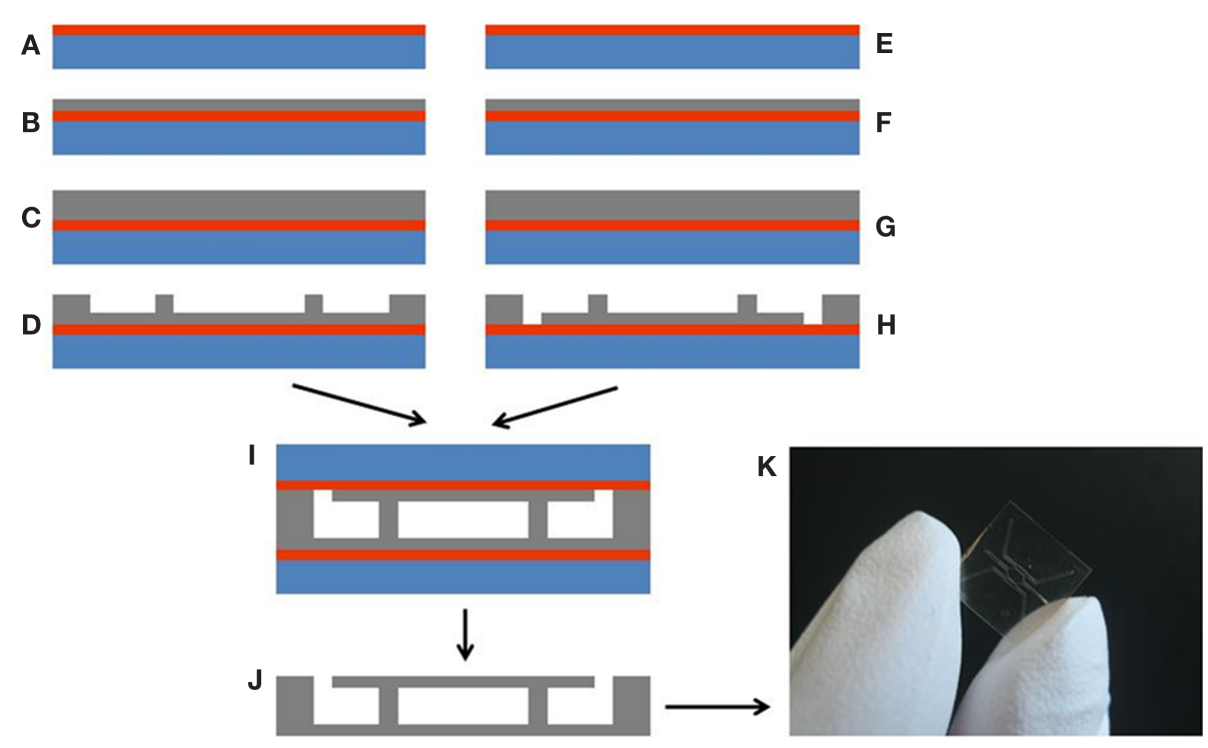

FIGURE 2 | Chip fabrication process: (A) Kapton film bonding to a Pyrex wafer, (B) processing of a $90 \mu \mathrm{m}$-thick SU-8 layer, (C) processing of chamber and microchannels layer, (D) wafer development, (E) Kapton film bonding to a
Pyrex wafer, (F) processing of a $90 \mu \mathrm{m}$-thick SU-8 layer, (G) processing of chamber and microchannels layer, (H) wafer development, (I) SU-8 to SU-8 bonding, (J) SU-8 device release, and (K) finished SU-8 microdevice. 


\section{Cell Culture}

MC3T3-E1 cells, mouse pre-osteoblasts (ATCC, CRL-2593, kindly donated by Doctor Izal from the University of Navarra, Spain), were routinely grown in $\alpha$-minimal essential media (MEM) (Lonza BE12-169) supplemented with 10\% v/v fetal calf serum (Sigma F7524) and penicillin/streptomycin (DE 17-602E). For three-dimensional cultures, all needed reagents, microdevices included, were placed on ice. Cells were trypsinized and resuspended in a calculated volume of medium ( $\alpha$-MEM supplemented with $10 \%$ fetal bovine serum) to reach the desired concentration of cells in the final hydrogel solution. About $100 \mu \mathrm{l}$ of hydrogel mixture (for a final collagen concentration of $1.2 \mathrm{mg} / \mathrm{ml}$ ) were prepared as follows: $17.25 \mu \mathrm{l}$ of sterile and distilled water; $12 \mu \mathrm{l}$ of DMEM 5X with L-glutamine (Sigma D5523); $0.75 \mu \mathrm{l}$ of $\mathrm{NaOH}$ $1 \mathrm{~N}$ (Sigma 221465); $30 \mu \mathrm{l}$ of collagen $4 \mathrm{mg} / \mathrm{ml}$ (Corning, 356236); and finally $40 \mu \mathrm{l}$ of cell suspension were added. If another collagen concentration was used, reagent amounts were modified accordingly. Using a chilled tip, solutions were injected into the device using a micropipette (detailed protocol in Section "Microdevice Design"). Then, droplets of 5-10 $\mu$ l were placed on top of each inlet to prevent hydrogel leakage and evaporation. Afterwards, the microfluidic device was placed into an incubator $\left(37^{\circ} \mathrm{C}\right.$ and $5 \%$ $\mathrm{CO}_{2}$ ) for $15 \mathrm{~min}$ to allow collagen polymerization. For use as a macroscopic control, $100 \mu \mathrm{l}$ of solution was allowed to polymerize on Petri dishes.

\section{Cell Viability}

Stock solutions of $5 \mathrm{mg} / \mathrm{ml}$ fluorescein diacetate (FDA) (Sigma F7378) and $2 \mathrm{mg} / \mathrm{ml}$ propidium iodide (PI) (Sigma P4170) were prepared following supplier instructions. To test cell viability within microfluidic devices and in Petri dishes, stock solutions of FDA and PI were diluted to 5 and $4 \mu \mathrm{g} / \mathrm{ml}$, respectively, in phosphate-buffered saline (PBS) (Lonza BE17-516F). Microdevices and Petri controls were washed once with PBS, and then filled with FDA/PI. Confocal images were immediately taken using a Nikon Eclipse Ti microscope. Images were collected at three different focal planes within each microdevice and Petri control. Experiments were repeated at least three times.

\section{Long-Term Cultures Under Restricted Conditions}

In order to study cell behavior during long-term experiments, cells were seeded into microfluidic devices at a concentration of 4 million cells $/ \mathrm{ml}$, using $1.2 \mathrm{mg} / \mathrm{ml}$ collagen hydrogel. Microdevices were incubated for $24 \mathrm{~h}$ in a $\mathrm{CO}_{2}$ incubator, and then placed inside the packaging tool. To enable medium flow to the packaging tool and microdevice assembly, the device was connected to a pressurized medium reservoir. A pressure regulator (Camozzi MC104-R20) was used to apply 0.1 bar and establish medium flow. Using a flow sensor (Sensirion LG16-0430), medium flow was monitored during experiments. Connections were made with fluorinated ethylene propylene (FEP) tubes (IDEX 1650 and 1684). A GL 45 screw cap (Schott Duran 11297 51) was placed onto the reservoir bottle along with corresponding connectors (Schott Duran 11298 14, Schott Duran 11298 15, Schott Duran 11562 92) that ensure good coupling of tubing. The outlet flow from the packaging tool was directed into a $15 \mathrm{ml}$ conical tube for collection of waste (Figure 3A). Flow data were recorded during
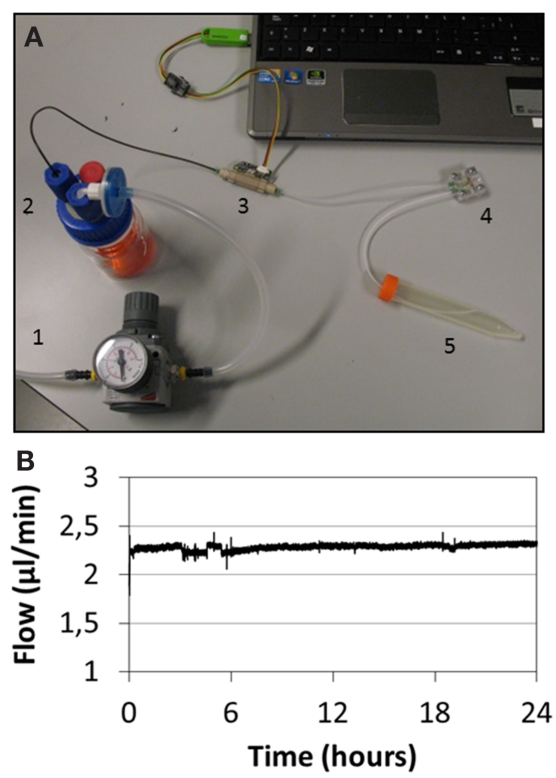

FIGURE 3 | (A) Microfluidic system. A pressure regulator (1) is connected to the medium reservoir (2), producing medium flow through the flow sensor (3) and packaging tool (4). System waste is collected in a tube (5). (B) Graph showing medium flow rate during an experiment.

experiments to verify flow stability (Figure 3B). Reservoir bottle culture medium was changed every 2 days to maintain stable concentration of media components. All the system was located within the incubator to maintain $37^{\circ} \mathrm{C}$ and $5 \% \mathrm{CO}_{2}$. Experiments were repeated three times.

\section{Image Analysis}

In order to analyze cell viability in different culture conditions, viable and dead cells were counted within the central microchamber using automated Fiji software (http://fiji.sc/Fiji). Due to the high cell densities reached in some microdevice regions during medium restriction experiments, cell counting algorithms were not reliable. In order to reduce error associated with experiments, we measured the area occupied by living or dead cells comparing live/dead occupied area distribution under restricted and unrestricted conditions. Data were analyzed using PSPP software, and statistical significance was set at $p<0.05$. Results are presented as mean \pm SE.

\section{Results}

\section{Microdevice Design}

In order to establish three-dimensional cell culture, microfluidic devices are usually designed with a central culture microchamber (including a dedicated inlet and outlet) and lateral microchannels at both sides that are delimited with a series of pillars (Figure 1). In this design, hydrogel, with or without cells embedded, should be confined within the culture chamber while lateral microchannels remain empty. To ensure unobstructed medium flow, it is important to avoid any hydrogel leakage from the microchamber into the lateral microchannels. During microdevice filling different interfaces appear between lateral pillars (50 $\mathrm{m}$ spacing distance) and 
one main interface across the central microchamber $(1000 \mu \mathrm{m}$ width). The liquid behavior during microdevice filling has been deduced from geometry of these interfaces and material properties using the Young-Laplace equation (Huang et al., 2009):

$$
\Delta P=\frac{\gamma}{R}
$$

where " $\Delta P$ " is the pressure difference between air and liquid, " $\gamma$ " is the liquid surface tension, and " $R$ " is the interface curvature radius. Within microfluidic devices, Eq. 1 can be rewritten as (Huang et al., 2009):

$$
\Delta P=-2 \gamma\left(\frac{\cos \theta}{W}+\frac{\cos \theta}{H}\right)
$$

Pressure difference at the interface is therefore expressed only as a function of the native contact angle between the liquid and solid $(\theta)$, the microchannel width $(W)$, and microchannel height $(H)$. Therefore, in hydrophilic materials $\left(\theta<90^{\circ}\right)$ such as SU$8\left(\theta\right.$ range between $72^{\circ}$ and $86^{\circ}$ ) (Schumacher et al., 2008;
Jokinen et al., 2012), this pressure difference is negative and spontaneous microdevice filling started (Figure 4A). When liquid spontaneously reached the central microchamber two lateral interfaces and one main front interface appeared, reaching a new interface curvature radius of 0 (Figure 4B). Under these circumstances capillary forces disappeared and interfaces got pinned, stopping microdevice filling. In order to stop this spontaneous filling, square-shape pillars were critical and showed a robust interface halting. To resume advancing external pressure had to be exerted, increasing the curvature radius of all interfaces until at least one of them reached a curvature equal to the native angle (Figure 4C). Due to their different geometry, when an external pressure was applied this curvature increase was lower on lateral interfaces compared to the main front interface. Therefore, main front interface reached first a curvature equal to the contact angle, resuming advancing trough central microchamber but without liquid leakage on lateral microchannels (Figure 4D). When design with such geometry and square shaped pillars, hydrogel confinement was achieved just locating a hydrogel droplet on the central inlet $(10 \mu \mathrm{l})$. Due to its inherent curvature, this droplet exerted the needed pressure to trigger fluid advancement through the

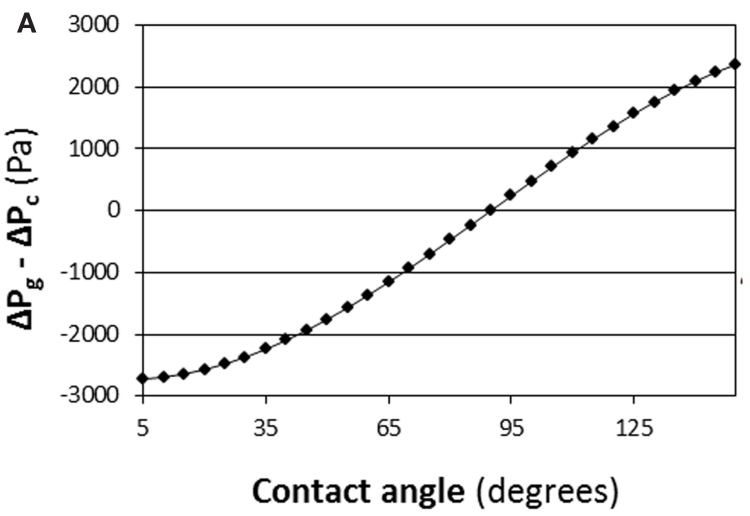

C
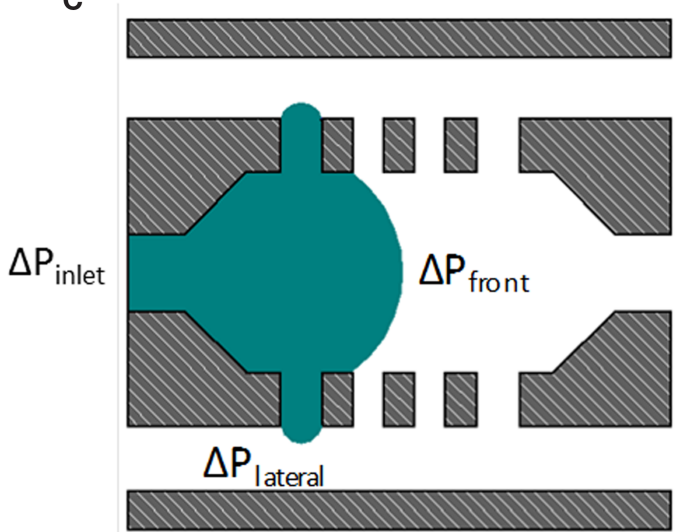

B

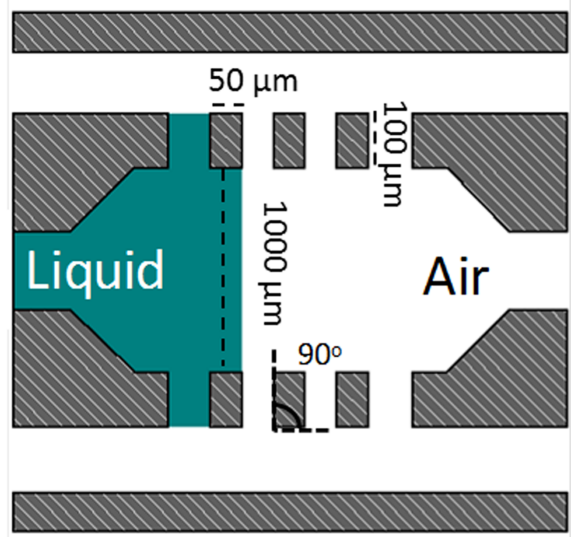

D
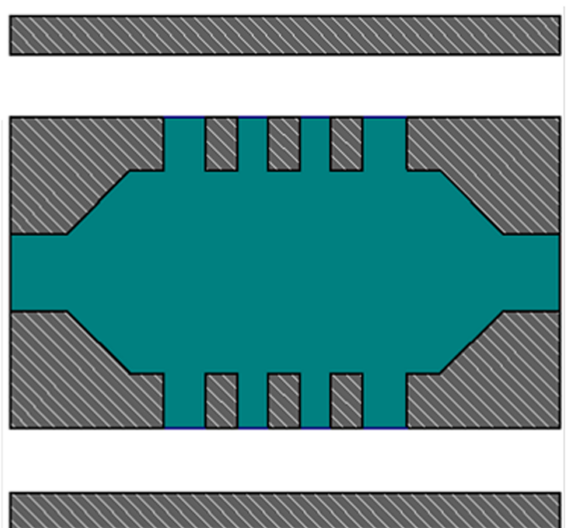

FIGURE 4 | (A) shows how the difference between $\Delta P_{\text {lateral }}$ and $\Delta P_{\text {front }}$ changes with the contact angle between the liquid and the material. In this model, water surface tension $\left(0.072 \mathrm{~N} / \mathrm{m}^{2}\right)$, gap distance $(50 \mu \mathrm{m})$, microchamber width (1000 $\mu \mathrm{m})$, and microdevice height $(300 \mu \mathrm{m})$ were kept constant, while contact angle ranged between $5^{\circ}$ and $150^{\circ}$ in $5^{\circ}$ steps. (B-D) shows the confinement process within hydrophilic SU-8-based microdevices. Capillary valves are created at the posts due to the $90^{\circ}$ change in post wall direction, illustrated in (B). Lateral capillary valves can support a higher pressure than the central one $\left(\Delta \mathrm{P}_{\text {lateral }}>\Delta \mathrm{P}_{\text {front }}\right)$. This configuration allows hydrogel confinement within a hydrophilic microdevice, as shown in (D). 
central microchamber, but not on lateral channels. Blue-colored water and either gelatine ( 1 and $4 \mathrm{mg} / \mathrm{ml}$ ) or collagen $(1.2$ and $2.4 \mathrm{mg} / \mathrm{ml}$ ) hydrogel was used to fill the microdevice employing the above-described method, and resulted in no leakage into lateral microchannels (Figure 5A). Once hydrogel, with cells embedded, was polymerized, additional solutions could be introduced to the microdevice through one or both lateral microchannels without any mixing except diffusion (Figure 5B).

\section{Influence of Culture Conditions on Cell Viability}

Biocompatibility of microdevices was evaluated using different cellular and collagen densities. Cell densities of $1 \times 10^{6}, 3 \times 10^{6}$, and $10 \times 10^{6}$ cells $/ \mathrm{ml}$ in collagen hydrogels of 1.2 and $2.4 \mathrm{mg} / \mathrm{ml}$ were tested. Cell viability for each condition was evaluated within microfluidic devices as well as in macroscopic controls on cell culture dishes. After $24 \mathrm{~h}$, cell viability was evaluated by FDA/PI labeling. All culture conditions yielded viability higher than $90 \%$ (Figure 6), and similar values were obtained for both microdevices and macroscopic controls. In these experiments, measurements were made after $24 \mathrm{~h}$ in culture to minimize possible nutrient starvation within the microdevice. Each condition was tested at least three times. Taken together with the aforementioned design considerations, these results support the use of SU-8 as suitable material for the development of three-dimensional cell culture microfluidic devices.

\section{Self-Induced Chemotaxis Under Restricted Conditions}

Cells are capable to adapt their behavior in response to their own microenvironment modifications. To investigate this notion, $1.2 \mathrm{mg} / \mathrm{ml}$ collagen hydrogel holding 4 million cells $/ \mathrm{ml}$ was confined into the central microchamber. Microfluidic devices were left in static conditions on a Petri dish for $24 \mathrm{~h}$ to allow cell attachment and spreading within the hydrogel. One microdevice was then enclosed in a packaging tool, and all inlets were sealed with the exception of one pair to allow medium perfusion through one lateral microchannel (restricted conditions). After medium flow was enabled, the system was incubated for 7 days. The other microdevice was left on the Petri dish and media was refreshed once a day through both lateral microchannels (unrestricted conditions). Post-incubation images of cell distribution within the culture microchamber show cell distribution in the different conditions (Figure 7). Cell distribution just after hydrogel confinement was apparently homogeneous across the entire microchamber (Figure 7A). After 1 week in culture under unrestricted conditions cell distribution seemed to remain unchanged, showing a homogeneous cell distribution (Figure 7B). However, under restricted conditions the result was completely different, showing an asymmetric cell distribution within the central microchamber (Figure 7C). Under restricted condition cells seemed to concentrate nearby to the perfused lateral microchannel. This asymmetric distribution under restricted conditions could be explained by an asymmetric proliferation or asymmetric mortality within the central microchamber or also by a migratory process. To address this question, the taken images in the different conditions were split vertically into two symmetric halves and area occupied by living and dead cells was analyzed (Figure 8). As expected, viable cells distribution after hydrogel injection (0 days) and after 7 days under unrestricted condition was nearly identical in both halves. On the other hand, after 7 days under restricted conditions, cell distribution dramatically changed. In this case, the area occupied by viable cells in the microchamber half closer to the nutrient source was significantly higher compared to the other half ( $p$-value $<0.05)$. Additionally, the total area occupied by live cells in all the microchambers remained almost constant between the different conditions ( $p$-value $>0.05$ ). This suggests that there was no cell proliferation under restricted or unrestricted conditions. Furthermore, the area occupied by dead cells remained also constant in all conditions, showing medium restriction caused any cell mortality ( $p$-value $>0.05$ ). Taking together, these results demonstrated that under restricted conditions cell metabolism generated a medium depletion, which led to a self-induced chemotactic process toward the nutrient source.

\section{Discussion}

When working with microdevices, material properties play a critical role during the critical step of liquid filling, as it has been described previously. In most of the microsystems reported in literature made of PDMS including pillars, hydrogel confinement is achieved by taking advantage of the substrate inherent hydrophobicity. Due to this hydrophobicity, interface pressure barriers are always positive, allowing the use external pressure with the pipette to push the hydrogel within the microdevice. As liquid injection
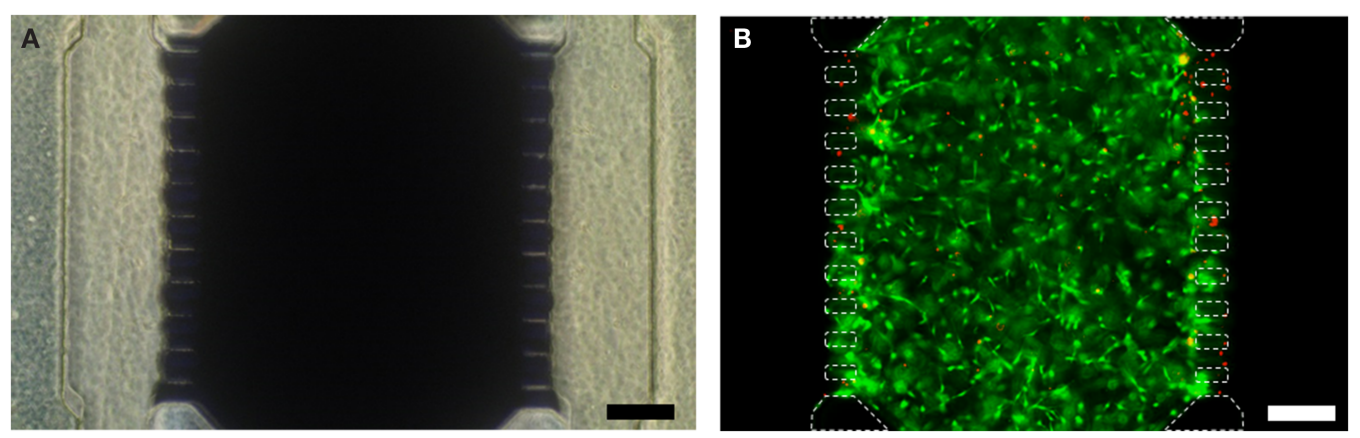

FIGURE $\mathbf{5}$ | (A) Blue-colored water is confined in the central microchamber with no leakage observed into lateral microchannels. (B) Fluorescence image of cells $\left(4 \times 10^{6} \mathrm{cells} / \mathrm{ml}\right)$ embedded in a collagen hydrogel $(1.2 \mathrm{mg} / \mathrm{ml})$ confined for $24 \mathrm{~h}$. Viable cells are shown in green while dead cells are in red. Scale bar is $200 \mu \mathrm{m}$. 
A

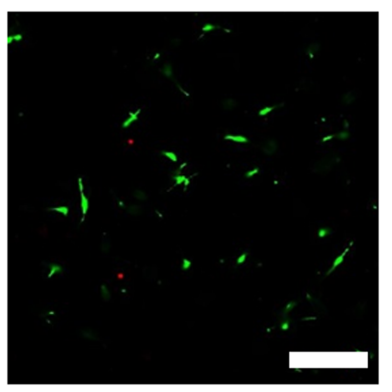

B

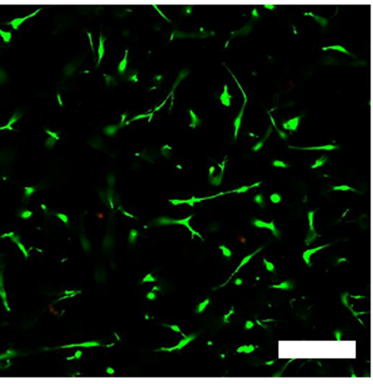

c

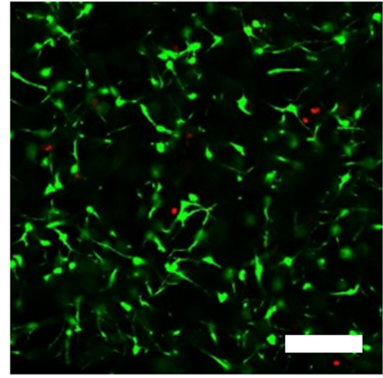

D

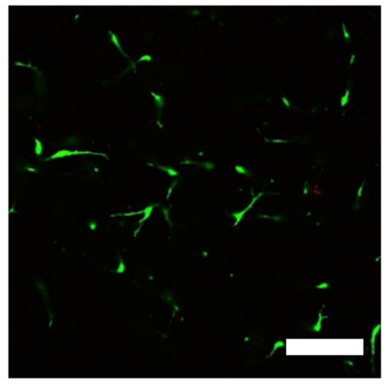

E

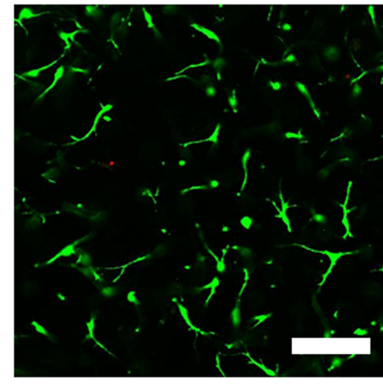

$F$

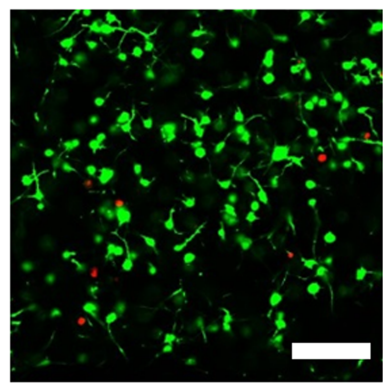

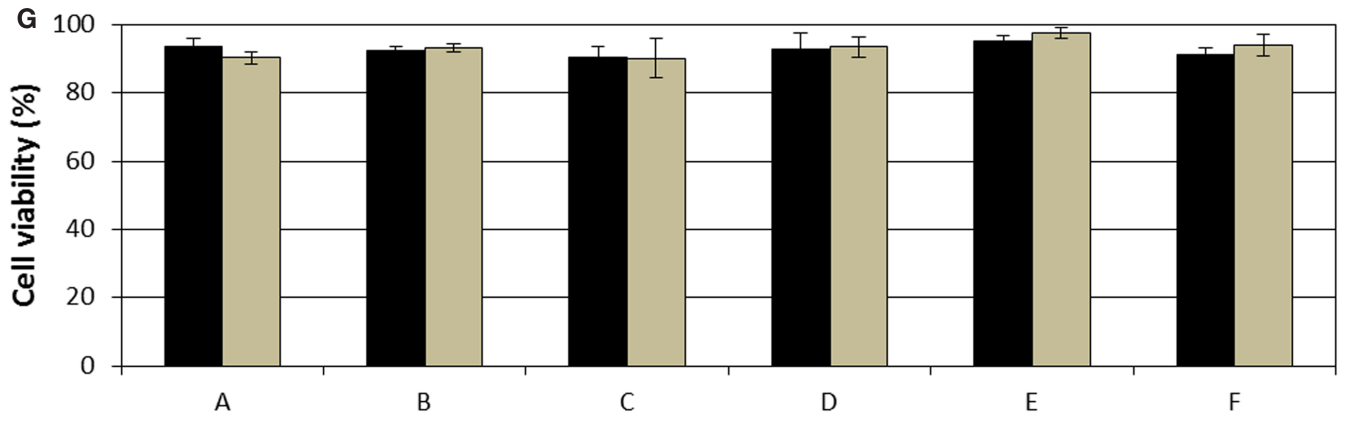

FIGURE 6 | Cell viability at $1 \times 10^{6}, 3 \times 10^{6}$, and $10 \times 10^{6} \mathrm{cells} / \mathrm{ml}$ in $1.2 \mathrm{mg} / \mathrm{ml}$ collagen hydrogels is shown in $(A-C)$, respectively. Cell viability at $1 \times 10^{6}, 3 \times 10^{6}$, and $10 \times 10^{6} \mathrm{cell} / \mathrm{ml}$ in $2.4 \mathrm{mg} / \mathrm{ml}$ collagen hydrogels is shown in (D-F), respectively. Scale bar is $100 \mu \mathrm{m}$.
(G) Graph shows cell viability analysis under (A-F) culture conditions within the microdevice and on a Petri dish. No statistical difference ( $p$-value $>0.05$ ) was found between microdevices and hydrogels on Petri dishes.
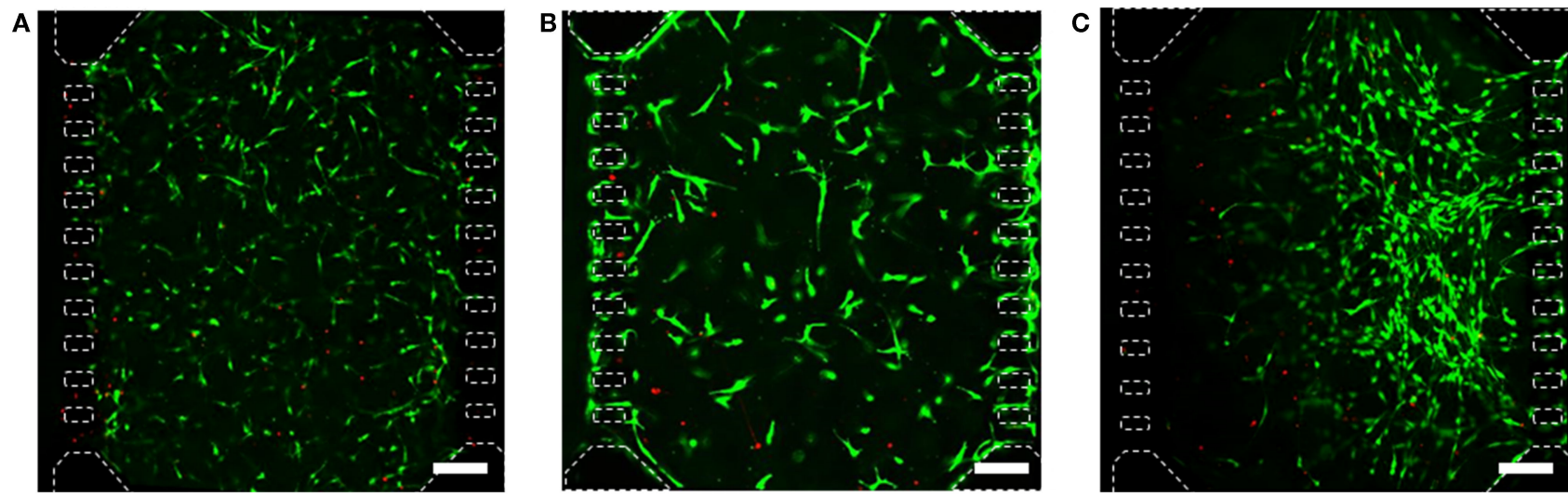

FIGURE 7 | Confocal images showing cell viability and distribution for $4 \times 10^{6}$ cells $/ \mathrm{ml}$ in $1.2 \mathrm{mg} / \mathrm{ml}$ collagen hydrogel. (A) Cell distribution after hydrogel injection. (B) Cell distribution after 7 days in unrestricted conditions. (C) Cell distribution in restricted conditions, medium flow was enabled only through the right microchannel. Scale bar is $200 \mu \mathrm{m}$. 

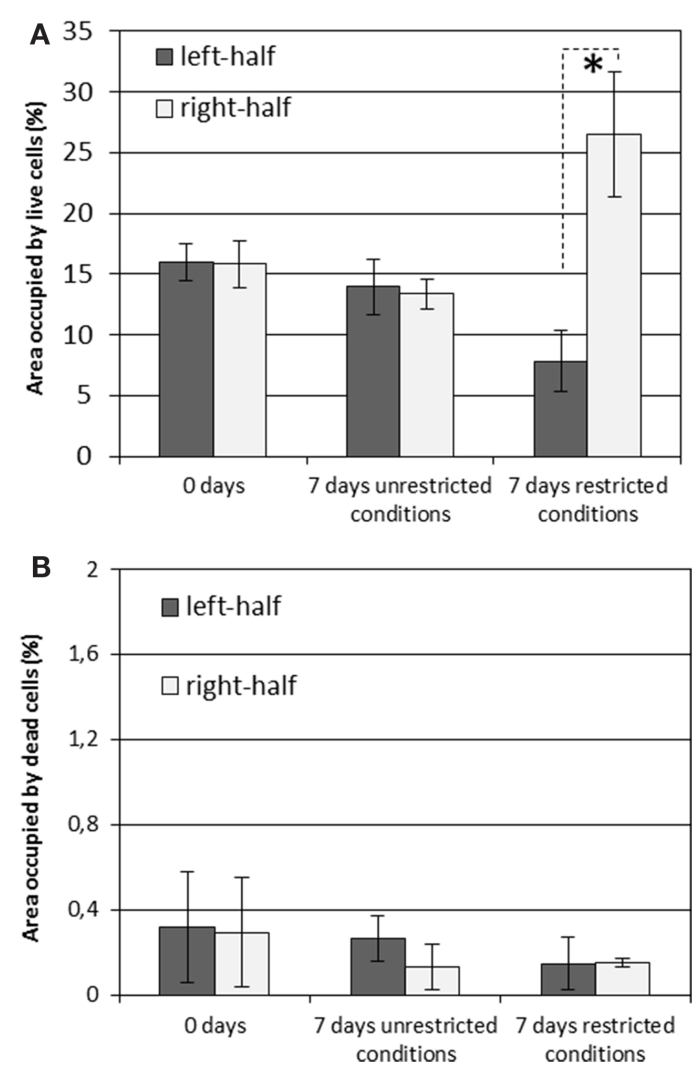

FIGURE 8 | Area occupied by viable (A) and dead (B) cells in the two central microchamber halves. When cells were seed, cell distribution was equal in both microchamber halves. After 7 days in unrestricted conditions, cell distribution remained constant in both halves. When medium flow was enabled through only right lateral microchannel, restricted conditions, the distribution of viable cells was clearly asymmetric, being most of cells concentrated close to the medium source $\left({ }^{*} p\right.$-value $\left.<0.05\right)$. Dead cell distribution barely changed in all circumstances.

through wider channels require lower pressure compared to narrower ones in hydrophobic microdevices, the hydrogel will preferably fill the central chamber rather than blocking the lateral microchannels protected by the micropillars. Although PDMSbased microdevices are normally rendered hydrophilic in order to covalently bond them with glass coverslips or between two separated PDMS layers, this change is reversible. After some time (commonly from few hours up to few days), PDMS hydrophobicity is recovered and hydrogel confinement using pillars is achieved as explained above (Huang et al., 2009). However, in hydrophilic materials (contact angle is lower than $90^{\circ}$ ), pressure barriers have negative values, favoring spontaneous filling of narrower microchannels. Therefore, pillars on hydrophilic microdevices are unable to prevent leakage as they do in PDMS microdevices and specific changes on pillar shape must be performed.

The design presented in this paper represents an alternative strategy for microdevice filling based on geometric configurations known as capillary valves (Leu and Chang, 2004; Cho et al., 2007; Zimmermann et al., 2007; Chen et al., 2008). These valves are created by an abrupt change in microdevice wall direction. When interface reaches this abrupt change in wall direction, curvature radius becomes 0 , and spontaneous filling is stopped. In order to design capillary valves in hydrophilic microdevices, the change in pillar wall direction $(\beta)$ needs to satisfy $\beta \geq 90^{\circ}-\theta$ (for SU-8, $\beta \geq 18^{\circ}$ ). As $\beta$ increases, capillary valves can withstand higher pressures before failure (in our square shaped pillars $\beta=90^{\circ}$ ). In principle, the main front interface created across the central microchamber could be halted at the first series of pillars, since they create also a capillary valve. Due to its larger width, however, lower external pressure is required to reach a curvature equal to the native angle $\left(\mathrm{P}_{\text {front }}\right)$ as compared to the lateral interface $\left(\mathrm{P}_{\text {lateral }}\right)$ (Safavieh and Juncker, 2013). As long as the pressure applied to the inlet $\left(\mathrm{P}_{\text {inlet }}\right)$ is kept between these two values (i.e., $\left.\mathrm{P}_{\text {front }}<\mathrm{P}_{\text {inlet }}<\mathrm{P}_{\text {lateral }}\right)$, microchamber filling will occur without leakage into lateral microchannels. When a SU- 8 based microdevice is designed with the described geometry, location of a droplet $(\approx 10 \mu \mathrm{l})$ exerted a pressure that spanned between these ranges, allowing proper hydrogel confinement.

The microdevice presented in this article has been then tested on self-induced chemotaxis experiments. In contrast with previous reported chemotaxis experiments, the nutrient chemotaxis is created by the activity of cells, a situation is to be considered closer to natural gradient environments. During the course of the experiment, metabolism of cells located close to the perfused channel created a nutrient starvation for cells located far away, which led to a strong migratory process toward the perfused lateral microchannel. Interestingly, in this long-term culture, experiments cells seemed to enclose around SU-8 pillars. Proliferation rate reduction was quite expected since many reports have demonstrated how cell proliferation is normally lower in 3D systems compared with 2D cultures (Smalley et al., 2006; Wells, 2008; Ali et al., 2014; Antoni et al., 2015). Furthermore, an inverse relationship between the collagen hydrogel stiffness and proliferation rate has been shown in bone cells (Jha et al., 2014). Previous results obtained in imposed chemotactic assays show that cell migration occurs after few minutes or hours (Zicha et al., 1997; Zimmermann et al., 2007; Bhattacharjee et al., 2010; Kim et al., 2013b, 2010; Chen et al., 2011; Funamoto et al., 2012). In contrast, in the presented paper, a more complex response was observed, since cell response occurred only after several days under nutrient restriction. We hypothesize that at first there was enough amount of nutrients within the central microchamber, and only after few days this concentration dropped below a threshold, which impelled the cells to migrate. As a consequence, the process for the creation and establishment of nutrient starvation and cell reaction could be better studied in future experiments using the developed microsystem and protocol. While modifying initial cell density or specific nutrient concentrations (glucose, etc.) within the central microchamber could lead to different responses and even to the generation of cellular autophagy and necrotic regions.

\section{Conclusion}

In this paper, we present a designed based protocol for hydrogel confinement within microfluidic devices made of hydrophilic materials. Therefore, it can be suitable for any hydrophilic material by a simple adjustment of the chamber design, facilitating the use 
of new materials such as polystyrene, COC, or SU-8. The developed protocol was tested with a hydrophilic SU-8-based microdevice, and produced positive results independent of the injected solution. After hydrogel confinement, biocompatibility of the microdevice was confirmed, showing cell viabilities $>90 \%$ in all cases. Under restricted conditions, and due to cell metabolism, a strong migratory process toward the nutrient source was induced. Many other reports have shown previously examples of chemotaxis, but in such articles chemotaxis conditions are externally imposed. This is the first example in which the chemotaxis is generated naturally by cells within a microfluidic device, causing a self-induced migratory process. This self-induced process showed a dynamic very different compared with other imposed chemotaxis assays. Thus, combining the described methodology, with sensor integration within the SU-8 based microdevices, precise

\section{References}

Ali, M. Y., Chuang, C. Y., and Saif, M. T. (2014). Reprogramming cellular phenotype by soft collagen gels. Soft Matter 10, 8829-8837. doi:10.1039/c4sm01602e

Altuna, A., Menendez de la Prida, L., Bellistri, E., Gabriel, G., Guimerà, A., Berganzo, J., et al. (2012). SU-8 based microprobes with integrated planar electrodes for enhanced neural depth recording. Biosens. Bioelectron. 37, 1-5. doi:10.1016/j.bios.2012.03.039

Altuna, A., Bellistri, E., Cid, E., Aivar, P., Gal, B., Berganzo, J., et al. (2013). SU-8 based microprobes for simultaneous neural depth recording and drug delivery in the brain. Lab. Chip 13, 1422-1430. doi:10.1039/c3lc41364k

Antoni, D., Burckel, H., Josset, E., and Noel, G. (2015). Three-dimensional cell culture: a breakthrough in vivo. Int. J. Mol. Sci. 16, 5517-5527. doi:10.3390/ ijms 16035517

Baker, B. M., and Chen, C. S. (2012). Deconstructing the third dimension: how 3D culture microenvironments alter cellular cues. J. Cell. Sci. 125, 3015-3024. doi:10.1242/jcs.079509

Bhattacharjee, N., Li, N., Keenan, T. M., and Folch, A. (2010). A neuron-benign microfluidic gradient generator for studying the response of mammalian neurons towards axon guidance factors. Integr. Biol. (Camb) 2, 669-679. doi:10.1039/ c0ib00038h

Blanco, F., Agirregabiria, M., Garcia, J., Berganzo, J., Tijero, M., and Arroyo, M. (2004). Novel three-dimensional embedded SU-8 microchannels fabricated using a low temperature full wafer adhesive bonding. J. Micromech. Microeng. 14, 1047-1056. doi:10.1088/0960-1317/14/7/027

Breslin, S., and O'Driscoll, L. (2013). Three-dimensional cell culture: the missing link in drug discovery. Drug Discov. Today 18, 240-249. doi:10.1016/j.drudis. 2012.10.003

Calvo, V., Ezkerra, A., Elizalde, J., Fernández, L., Berganzo, J., Mayora, K., et al. (2011). A highly integrated vertical SU8 valve for stepwise in-series reactions. J. Micromech. Microeng. 21, 065037. doi:10.1088/0960-1317/21/6/065037

Castaño-Álvarez, M., Fernández-Abedul, M. T., Costa-García, A., Agirregabiria, M., Fernández, L., Ruano-López, J., et al. (2009). Fabrication of SU-8 based microchip electrophoresis with integrated electrochemical detection for neurotransmitters. Talanta 80, 24-30. doi:10.1016/j.talanta.2009.05.049

Chen, J., Huang, P., and Lin, M. (2008). Analysis and experiment of capillary valves for microfluidics on a rotating disk. Microfluid. Nanofluidics 4, 427-437. doi:10.1007/s10404-007-0196-x

Chen, Y. A., King, A. D., Shih, H. C., Peng, C. C., Wu, C. Y., Liao, W. H., et al. (2011). Generation of oxygen gradients in microfluidic devices for cell culture using spatially confined chemical reactions. Lab. Chip 11, 3626-3633. doi:10. 1039/c1lc20325h

Cho, H., Kim, H., Kang, J., and Kim, T. (2007). How the capillary burst microvalve works. J. Colloid Interface Sci. 306, 379-385. doi:10.1016/j.jcis.2006. 10.077

Duval, D., González-Guerrero, A., Dante, S., Osmond, J., Monge, R., Fernández, L., et al. (2012). Nanophotonic lab-on-a-chip platforms including novel bimodal interferometers, microfluidics and grating couplers. Lab. Chip 12, 1987-1994. doi: $10.1039 / \mathrm{c} 2 \mathrm{lc} 40054 \mathrm{e}$ monitoring of metabolites could be done in real time during biological process like tumor growth and invasion.

\section{Author Contributions}

Conception and design: IO and LF. Protocol and methodology development: JA, RM, GL, MM, MA, JB, IO, and LF. Acquisition data: JA, MM, and GL. Manuscript writing and reviewing: JA, RM, $\mathrm{IO}, \mathrm{LF}$, and MD.

\section{Acknowledgments}

This work has been supported by the National Research Program of Spain, project “dpi2011-28262-c04-01" and "bes-2012-059940." JA studentship was provided by the Aragon government.

El-Ali, J., Sorger, P. K., and Jensen, K. F. (2006). Cells on chips. Nature 442, 403-411. doi:10.1038/nature05063

Ezkerra, A., Fernández, L., Mayora, K., and Ruano-López, J. (2007). Fabrication of SU-8 free-standing structures embedded in microchannels for microfluidic control. J. Micromech. Microeng. 17, 2264-2271. doi:10.1088/0960-1317/17/11/013

Ezkerra, A., Fernandez, L. J., Mayora, K., and Ruano-Lopez, J. M. (2011). SU8 diaphragm micropump with monolithically integrated cantilever check valves. Lab. Chip 11, 3320-3325. doi:10.1039/c1lc20324j

Falasca, M., Raimondi, C., and Maffucci, T. (2011). Boyden chamber. Methods Mol. Biol. 769, 87-95. doi:10.1007/978-1-61779-207-6_7

Farahat, W. A., Wood, L. B., Zervantonakis, I. K., Schor, A., Ong, S., Neal, D., et al. (2012). Ensemble analysis of angiogenic growth in three-dimensional microfluidic cell cultures. PLoS ONE 7:e37333. doi:10.1371/journal.pone.0037333

Fernández, L., Altuna, A., Tijero, M., Gabriel, G., Villa, R., Rodríguez, M., et al. (2009). Study of functional viability of SU-8-based microneedles for neural applications. J. Micromech. Microeng. 19, 025007. doi:10.1088/0960-1317/19/2/ 025007

Franco, S. J., and Muller, U. (2011). Extracellular matrix functions during neuronal migration and lamination in the mammalian central nervous system. Dev. Neurobiol. 71, 889-900. doi:10.1002/dneu.20946

Funamoto, K., Zervantonakis, I. K., Liu, Y., Ochs, C. J., Kim, C., and Kamm, R. D. (2012). A novel microfluidic platform for high-resolution imaging of a threedimensional cell culture under a controlled hypoxic environment. Lab. Chip 12, 4855-4863. doi:10.1039/c2lc40306d

Gerhardt, H., and Betsholtz, C. (2005). "How do endothelial cells orientate?" in Angiogenesis mechanisms, Chap. 1. eds M. Clauss and G. Breier (Switzerland: Birkhäuser Basel), 3-15. doi:10.1007/3-7643-7311-3_1

González, I., Fernández, L., Gómez, T., Berganzo, J., Soto, J., and Carrato, A. (2012) A polymeric chip for micromanipulation and particle sorting by ultrasounds based on a multilayer configuration. Sens. Actuators B Chem. 144, 310-317. doi:10.1016/j.snb.2009.10.042

Guo, X., Elliott, C. G., Li, Z., Xu, Y., Hamilton, D. W., and Guan, J. (2012). Creating 3D angiogenic growth factor gradients in fibrous constructs to guide fast angiogenesis. Biomacromolecules 13, 3262-3271. doi:10.1021/bm301029a

Huang, C. P., Lu, J., Seon, H., Lee, A. P., Flanagan, L. A., Kim, H. Y., et al. (2009). Engineering microscale cellular niches for three-dimensional multicellular cocultures. Lab. Chip 9, 1740-1748. doi:10.1039/b818401a

Huang, G., Wang, L., Wang, S., Han, Y., Wu, J., Zhang, Q., et al. (2012). Engineering three-dimensional cell mechanical microenvironment with hydrogels. Biofabrication 4, 042001. doi:10.1088/1758-5082/4/4/042001

Jeon, J. S., Chung, S., Kamm, R. D., and Charest, J. L. (2011). Hot embossing for fabrication of a microfluidic 3D cell culture platform. Biomed. Microdevices 13, 325-333. doi:10.1007/s10544-010-9496-0

Jha, A. K., Jackson, W. M., and Healy, K. E. (2014). Controlling osteogenic stem cell differentiation via soft bioinspired hydrogels. PLoS ONE 9:e98640. doi:10.1371/ journal.pone.0098640

Jokinen, V., Suvanto, P., and Franssila, S. (2012). Oxygen and nitrogen plasma hydrophilization and hydrophobic recovery of polymers. Biomicrofluidics 6, 16501-1650110. doi:10.1063/1.3673251 
Keenan, T. M., and Folch, A. (2008). Biomolecular gradients in cell culture systems. Lab. Chip 8, 34-57. doi:10.1039/B711887B

Kim, D. H., Hossain, M. A., Kim, M. Y., Kim, J. A., Yoon, J. H., Suh, H. S., et al. (2013a). A novel resveratrol analogue, HS-1793, inhibits hypoxia-induced HIFlalpha and VEGF expression, and migration in human prostate cancer cells. Int. J. Oncol. 43, 1915-1924. doi:10.3892/ijo.2013.2116

Kim, B. J., Hannanta-anan, P., Chau, M., Kim, Y. S., Swartz, M. A., and Wu, M. (2013b). Cooperative roles of SDF-1alpha and EGF gradients on tumor cell migration revealed by a robust 3D microfluidic model. PLoS ONE 8:e68422. doi:10.1371/journal.pone.0068422

Kim, S., Kim, H. J., and Jeon, N. L. (2010). Biological applications of microfluidic gradient devices. Integr. Biol. (Camb) 2, 584-603. doi:10.1039/c0ib00055h

Korpos, E., Wu, C., Song, J., Hallmann, R., and Sorokin, L. (2010). Role of the extracellular matrix in lymphocyte migration. Cell Tissue Res. 339, 47-57. doi:10. 1007/s00441-009-0853-3

Kothapalli, C. R., van Veen, E., de Valence, S., Chung, S., Zervantonakis, I. K., Gertler, F. B., et al. (2011). A high-throughput microfluidic assay to study neurite response to growth factor gradients. Lab. Chip 11, 497-507. doi:10.1039/ c0lc00240b

Kotzar, G., Freas, M., Abel, P., Fleischman, A., Roy, S., Zorman, C., et al. (2002). Evaluation of MEMS materials of construction for implantable medical devices. Biomaterials 23, 2737-2750. doi:10.1016/S0142-9612(02)00007-8

Lesher-Perez, S. C., Frampton, J. P., and Takayama, S. (2013). Microfluidic systems: a new toolbox for pluripotent stem cells. Biotechnol. J. 8, 180-191. doi:10.1002/ biot.201200206

Leu, T., and Chang, P. (2004). Pressure barrier of capillary stop valves in micro sample separators. Sens. Actuators A 115, 508-515. doi:10.1016/j.sna.2004.02.036

Lorenz, H., Despont, M., Fahrni, M., LaBianca, N., Vettiger, P., and Renaud, P. (1997). SU-8: a low-cost negative resist for MEMS. J. Micromech. Microeng. 7, 121-124. doi:10.1088/0960-1317/7/3/010

Mousseau, Y., Leclers, D., Faucher-Durand, K., Cook-Moreau, J., Lia-Baldini, A. S., Rigaud, M., et al. (2007). Improved agarose gel assay for quantification of growth factor-induced cell motility. Biotechniques 43, 509-516. doi:10.2144/000112557

Muinonen-Martin, A. J., Veltman, D. M., Kalna, G., and Insall, R. H. (2010). An improved chamber for direct visualisation of chemotaxis. PLoS ONE 5:e15309. doi:10.1371/journal.pone.0015309

Nagelkerke, A., Bussink, J., Mujcic, H., Wouters, B. G., Lehmann, S., Sweep, F. C., et al. (2013). Hypoxia stimulates migration of breast cancer cells via the PERK/ATF4/LAMP3-arm of the unfolded protein response. Breast Cancer Res. 15, R2. doi:10.1186/bcr3373

Nemani, K. V., Moodie, K. L., Brennick, J. B., Su, A., and Gimi, B. (2013). In vitro and in vivo evaluation of SU-8 biocompatibility. Mater. Sci. Eng. C Mater. Biol. Appl. 33, 4453-4459. doi:10.1016/j.msec.2013.07.001

Ni, M., Tong, W. H., Choudhury, D., Rahim, N. A., Iliescu, C., and Yu, H. (2009). Cell culture on MEMS platforms: a review. Int. J. Mol. Sci. 10, 5411-5441. doi:10.3390/ijms10125411

Nieto, M., Frade, J. M., Sancho, D., Mellado, M., Martinez-A, C., and SanchezMadrid, F. (1997). Polarization of chemokine receptors to the leading edge during lymphocyte chemotaxis. J. Exp. Med. 186, 153-158. doi:10.1084/jem.186. 1.153

Noman, M. Z., Messai, Y., Carre, T., Akalay, I., Meron, M., Janji, B., et al. (2011). Microenvironmental hypoxia orchestrating the cell stroma cross talk, tumor progression and antitumor response. Crit. Rev. Immunol. 31, 357-377. doi:10. 1615/CritRevImmunol.v31.i5.10

Page, H., Flood, P., and Reynaud, E. G. (2013). Three-dimensional tissue cultures: current trends and beyond. Cell Tissue Res. 352, 123-131. doi:10.1007/ s00441-012-1441-5

Raines, E. W. (2000). The extracellular matrix can regulate vascular cell migration, proliferation, and survival: relationships to vascular disease. Int. J. Exp. Pathol. 81, 173-182. doi:10.1046/j.1365-2613.2000.00155.x
Rigat-Brugarolas, L. G., Elizalde-Torrent, A., Bernabeu, M., De Niz, M., MartinJaular, L., Fernandez-Becerra, C., et al. (2014). A functional microengineered model of the human splenon-on-a-chip. Lab. Chip 14, 1715-1724. doi:10.1039/ c3lc51449h

Roussos, E. T., Condeelis, J. S., and Patsialou, A. (2011). Chemotaxis in cancer. Nat. Rev. Cancer 11, 573-587. doi:10.1038/nrc3078

Safavieh, R., and Juncker, D. (2013). Capillarics: pre-programmed, self-powered microfluidic circuits built from capillary elements. Lab. Chip 13, 4180-4189. doi:10.1039/c3lc50691f

Savino, W., Mendes-Da-Cruz, D. A., Smaniotto, S., Silva-Monteiro, E., and VillaVerde, D. M. (2004). Molecular mechanisms governing thymocyte migration: combined role of chemokines and extracellular matrix. J. Leukoc. Biol. 75, 951-961. doi:10.1189/jlb.1003455

Schumacher, J., Grodrian, A., Kremin, C., Hoffmann, M., and Metze, J. (2008). Hydrophobic coating of microfluidic chips structured by SU-8 polymer for segmented flow operation. J. Micromech. Microeng. 5, 055019. doi:10.1088/ 0960-1317/18/5/055019

Smalley, K. S., Lioni, M., and Herlyn, M. (2006). Life isn't flat: taking cancer biology to the next dimension. In vitro Cell. Dev. Biol. Anim. 42, 242-247. doi:10.1290/ 0604027.1

Song, J. W., Bazou, D., and Munn, L. L. (2012). Anastomosis of endothelial sprouts forms new vessels in a tissue analogue of angiogenesis. Integr. Biol. (Camb) 4 , 857-862. doi:10.1039/c2ib20061a

Torrejon, K. Y., Pu, D., Bergkvist, M., Danias, J., Sharfstein, S. T., and Xie, Y. (2013). Recreating a human trabecular meshwork outflow system on microfabricated porous structures. Biotechnol. Bioeng. 110, 3205-3218. doi:10.1002/bit. 24977

Verdoy, D., Barrenetxea, Z., Berganzo, J., Agirregabiria, M., Ruano-López, J., Marimón, J., et al. (2012). A novel real time micro PCR based point-of-care device for Salmonella detection in human clinical samples. Biosens. Bioelectron. 32, 259-265. doi:10.1016/j.bios.2011.12.032

Vilares, R., Hunter, C., Ugarte, I., Aranburu, I., Berganzo, J., Elizalde, J., et al. (2010). Fabrication and testing of a SU-8 thermal flow sensor. Sens. Actuators B Chem. 147, 411-417. doi:10.1016/j.snb.2010.03.054

Wells, R. G. (2008). The role of matrix stiffness in regulating cell behavior. Hepatology 47, 1394-1400. doi:10.1002/hep.22193

Zhang, Y., Liu, Q., Wang, F., Ling, E. A., Liu, S., Wang, L., et al. (2013). Melatonin antagonizes hypoxia-mediated glioblastoma cell migration and invasion via inhibition of HIF-1alpha. J. Pineal Res. 55, 121-130. doi:10.1111/jpi.12052

Zicha, D., Dunn, G., and Jones, G. (1997). Analyzing chemotaxis using the Dunn direct-viewing chamber. Methods Mol. Biol. 75, 449-457.

Zigmond, S. H. (1977). Ability of polymorphonuclear leukocytes to orient in gradients of chemotactic factors. J. Cell Biol. 75, 606-616. doi:10.1083/ jcb.75.2.606

Zimmermann, M., Hunziker, P., and Delamarche, E. (2007). Valves for autonomous capillary systems. Microfluid. Nanofluidics 5, 395-402. doi:10.1039/c2lc41083d

Conflict of Interest Statement: The authors declare that the research was conducted in the absence of any commercial or financial relationships that could be construed as a potential conflict of interest.

Copyright () 2015 Ayuso, Monge, Llamazares, Moreno, Agirregabiria, Berganzo, Doblaré, Ochoa and Fernández. This is an open-access article distributed under the terms of the Creative Commons Attribution License (CC BY). The use, distribution or reproduction in other forums is permitted, provided the original author(s) or licensor are credited and that the original publication in this journal is cited, in accordance with accepted academic practice. No use, distribution or reproduction is permitted which does not comply with these terms. 